\section{A note on Filbey and Gazzaniga's "Splitting the brain with reaction time"}

\author{
WALTER F. MCKEEVER and MAURICE D. HULING \\ Ventura County Mental Health Department, Ventura, Calif. 93003
}

A recent issue of this journal reported an interesting experiment by Filbey \& Gazzaniga (1969). A condensed version of the original was subsequently reprinted (Filbey \& Gazzaniga, 1970) in a widely circulated publication of the National Institute of Mental Health. Filbey and Gazzaniga implied that "splitting the normal brain with reaction time" was a new strategy conceivable only on the groundwork provided by the split-brain studies of Sperry and Gazzaniga, which the authors cited.

Actually, Poffenberger (1912) "split the normal brain with reaction time" 58 years ago. Poffenberger tested the hypothesis that reaction time should be longer when a visual stimulus was directed to one hemisphere and a response was required from the other hemisphere than when the same hemisphere both received the stimulus and initiated the response. The results provided clear-cut support for the hypothesis, showing that reaction time was 5-6 msec longer when transcallosal relay was necessary. Indeed, Poffenberger should be credited with having "split the normal brain with reaction time" 12 years before Byk ov (1924) performed the first split-brain experiment on a dog.

Transcallosal transmission time (TTT) was also measured behaviorally by Efron (1963a, b) who hy pothesized that judgments of simultaneity of the onsets of lights in the left and right visual fields were made in the left hemisphere. His results were consistent with the hypothesis and showed that, for lights of the same intensity, the left light had to precede the right by 2-6 msec in order to have them judged as simultaneous. Efron also showed that stimulus intensity appeared to be an important determinant of TTT.

More recently, Jeeves (1969), using reaction time measures, found TTTs on the order of 2-3 msec for normals, and much longer interhemispheric transfer times for persons with congenital agenesis of the corpus callosum.

It should also be noted that other behavioral techniques for demonstrating the functional asymmetry of the cerebral hemispheres have been studied by many investigators (see, for example, Kimura, 1966, 1967).
Finally, Filbey and Gazzaniga's conclusion, that they have established a base TTT against which either hemispheric processing or transfer times for more complicated tasks may be meaningfully compared, seems unwarranted. Poffenberger's, Efron's, and Jeeves's estimates of TTT are in agreement but differ considerably from the 30 - to $40-\mathrm{msec}$ value obtained by Filbey and Gazzaniga, despite the fact that the task complexity in all three experiments seems similar. One should be aware of these discrepancies and of the need for resolving them experimentally.

Variations of the Filbey and Gazzaniga procedures might be illuminating. It is not clear from their report why only trials from the last two of the five experimental days were analyzed. Also, the practice of
The brief articles appearing in Psychonomic Science cannot possibly contain a full discussion or review of each subject introduced. In the present case the suggested addition would have left other dimensions of the problem uncovered. For those who do not know the history, it is as follows. Up until the mid-50s, the majority view in psychobiology was that the corpus callosum played little or no important role in integrating sensory or motor information across the cerebral hemispheres. This was in large part due to the seemingly exhaustive studies of Akelaitis $(1941,1943,1944,1942)$ at the University of Rochester who studied some 26 cases of partial and complete callosal section. His inability to demonstrate any reliable deficits undercut dozens of earlier studies-Poffenberger's (1912) being but one. Lashley, after reviewing the literature at the time commented that the corpus callosum's only function seemed to be to hold the cerebral hemispheres together.

It was in this context that Sperry and Myers commenced their now classic work on split-brains. It has now informing $S$ s of their reaction times to all trials might better be avoided, since it allows the possibility of subtle inadvertent shaping of left-right reaction-time differences.

REFERENCES

BYKOV, $k$. Versuche an Hunden mit durchschneiden des Corpus Callosum. Zentrablatt Neurologische Psvchiatrie. 1924-1925, 39, 199

EFRON, R. The effect of handedness on the perception of simultaneity and temporal order. Brain, 1963a, 86, 261-284.

EFRON, R. The effect of stimulus intensity on the perception of simultaneity in right- and left-handed subjects. Brain, $1963 \mathrm{~b}, 86,285-294$

FILBEY R A \& GAZZANIGA II S Splitting the normal brain with reaction time. Psychonomic Science, 1969,17 , 335-336.

FILBEY, R. A., \& GAZZANIGA, I. S Splitting the normal brain with reaction time. MIental Health Digest $1970,2,15$.

JEEVES, II. A. A comparison of interhemispheric transmission times in acallosals and normals. Psychonomic Science, 1969, 16, 245-246.

KIMURA, D. Dual functional asymmetry of the brain in visual perception. Neuropsychologia, 1966, 4, 275-285.

KINIURA, D Functional asymmetry of the brain in dichotic listening. Cortex, 1967. 3, 163-178

POFFENBERGER, A. T. Reaction time to retinal stimulation with special reference to the time lost in conduction through nerve centers. Archives of Psychology (New York), 1912, No. 23.

\title{
Reply to McKeever and Huling
}

\author{
MICHAEL S. GAZZANIGA \\ New York University, New York, N.Y. 10003
}

been almost 20 years since their pioneering studies began to spell out the role of the callosum. Starting in the early $60 \mathrm{~s}$, Sperry and I had the opportunity to study in detail callosum-sectioned humans. The broad outlines of the earlier animal findings, enhancing the importance of the callosum in transferring sensory and motor information interhemispherically, were confirmed and extended. As as result, only recently has there been general agreement that the corpus callosum is the structure mainly responsible for interhemispheric transmission of sensory and motor information. It was this evolutionary process that generated the framework for the studies in question. Without the positive findings of the last 10 years, the idea of ascertaining the nature of the callosal code using lateralized stimuli and reaction time would never have been considered interesting or important.

At any rate McKeever and Huling miss the point. Poffenberger used manual responses in reaction time whereas we used verbal. Indeed, there 
is very little common ground in the experiments. Noreover, using a manual response of the kind he employed unnecessarily complicates the interpretation and most probably neutralizes the "TTT," for it is becoming apparent from a number of other studies that movements elicited from proximal muscle system give no difference, whereas responses requiring cerebral exclusively-individual finger control reveal "TT" $T$ " differences.

The idea of trying to figure out the callosal code by using reaction time is upon us. It may fade. It's not as easy as it sounds. Nonetheless, within the year there have been a number of papers on the subject (Moscovitch \& Catlin, 1970; Bradshaw \& Perriment, 1970; Klatzky ${ }^{1}$ ). These authors, using different responses, different stimuli, and different almost everything else, found differences in transmission time ranging from. 10 to 60 msec. Finally, I can only urge those interested in the approach to join in.

\section{REFERENCES}

AKELAITIS, A. $\mathbf{J}$. Studies on corpus callosum; higher visual functions in each homonymous field following complete section of corpus callosum. Archives of Neurological Psychiatry (Chicago), 1941, 45.788

\title{
The role of interference and
} trace decay in the retention
of a simple psychomotor task*

\author{
STEPHEN D. SOUTHALL $\dagger$ and KENNETH A. BLICK \\ University of Richmond, Richmond, Va. 23173
}

The purpose of the present study was to show whether interference theory and/or trace decay theory account for the forgetting found in motor short-term memory. One variable was the number of prior responses $(0$ to 6$)$ which the $S$ experienced on a linear slide apparatus; another variable was the length of the retention interval $(5,40$, and $75 \mathrm{sec})$. There were no significant effects due to retention interval, number of prior responses, or the interaction between retention interval and prior responses.

The role that interference plays in verbal short-term memory (STM) has been established for some time, but its

* This research was supported in part by a Faculty Research Grant from the University of Richmond.

+Now at the University of Virginia.
AKELAITIS, A. J. Studies on corpus callosum; study of language functions (tactile and visual, lexia and gxaphia) unilaterally following section of corpus callosum. Journal of Neuropathological \& Experimental Neurology, 1943, 2, 226

AKELAITIS, A. J. Study of gnosis, praxis, and language following section of corpus callosum and anterior commissure. Journal of Neurosurgery, 1944, 1 , 94

AKELAITIS, A. J., RISTEEN, A. W., HERREN, R. Y., \& VAN WAGENEN, W. P. Studies on corpus callosum; contribution to study of dyspraxia and Neurological Psychiatry (Chicago), 1942, 47,971 Laterality effects and choice reaction time in a unimanual two-finger task. Perception \& Psychophysics, 1970, 7, 185-188.

MOSCOVITCH, M., \& CATLIN, J. Interhemispheric transmission of information: Measurement in normal man. Psychonomic Science, 1970, 18, 211-213.

POFFENBERGER, A. T. Reaction time to retinal stimulation with special reference to the time lost in conduction through nerve centers. Archives of Psychology (New York), 1912, No. 23.

\section{NOTE}

1. Klatzky, R. L. Interhemispheric transfer of test stimulus representations in memory scanning. In preparation. apraxia of corpus callosum. Archives of

BRADSHAW, J. L., \& PERRIMENT, A. D.

trace decay theory, or a combination of the two best accounts for the forgetting shown in motor STM.

Adams \& Dijkstra (1966) examined the retention of a linear motor response in which the basic variables were length of retention interval and number of reinforcements or trials before recall. Absolute error was found to be positively related to length of retention interval, and consequently, Adams \& Dijkstra (1966) interpreted their results in terms of a rapidly decaying memory trace, which became increasingly stable with reinforcement. Stelmach ( 1969 a), using a simple lever-positioning task, employed the three independent variables of magnitude of movement, retention interval, and number of prior positioning responses. Absolute error was found to be positively related to the number of prior positioning responses and to the length of the retention interval, but the magnitude of the movement was found to be nonsignificant. He considered the role of proactive interference in his results but seemed to favor the decay of the memory traces as the best explanation. However, in a similar study, Stelmach (1969b) found that absolute errors at recall were inversely related to the similarity of prior responses and that forgetting was found to be an increasing function of the retention interval. Stelmach (1969b) noted that the results concerning the similarity of responses were in direct opposition to the findings usually observed in verbal tasks and were not consistent with the predictions from interference theory.

More recently, Pepper \& Herman (1970) performed a series of five experiments measuring the retention of the magnitude of the force of a knob which was pushed or pulled through the vertical dimension in an attempt to establish whether decay, interference, or a combination of the two was the cause of the forgetting shown in motor STM. Pepper \& Herman (1970), by application of a second force response during the retention interval, showed that interference effects, traceable to the interpolated task, can be demonstrated for motor STM. The role of trace decay and interference theory in the area of motor STM is still nebulous, and it was the purpose of the present experiment to establish precisely the role of trace decay theory and/or interference theory in motor STM.

role in motor STM has not yet been confirmed. Although there have been many studies of verbal STM (Conrad \& Hille, 1958; Keppel \& Underwood, 1962; Murdock, 1961; Peterson \& Peterson, 1959), there have been relatively few studies on short-term retention of motor responses. It is not yet clear whether interference theory.

\section{METHOD}

One hundred and five undergraduates from the University of Richmond participated in the experiment. Fifteen males and 20 females served in each of the three retention intervals 